NISSUNA UMANA INVESTIGAZIONE SI PUO DIMANDARE VERA SCIENZIA S'ESSA NON PASSA PER LE MATEMATICHE DIMOSTRAZIONI LEONARDO DA VINCI

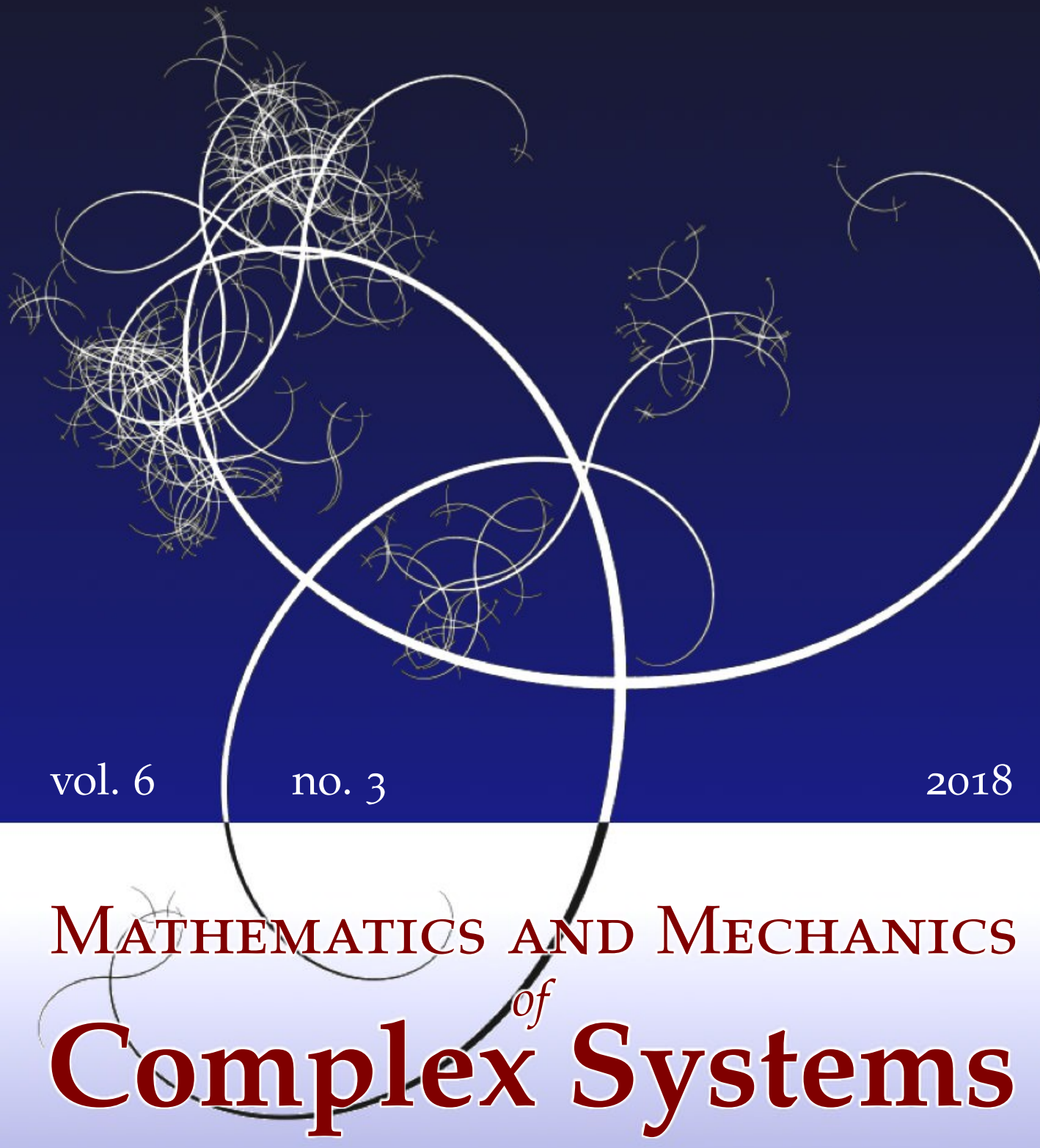

SANTOSH KumaR AND Dinbandhu MANDAL

GENERATION OF SH-TYPE WAVES DUE TO SHEARING STRESS DISCONTINUITY IN AN ANISOTROPIC LAYER OVERLYING AN INITIALLY STRESSED ELASTIC HALF-SPACE 


\title{
GENERATION OF SH-TYPE WAVES DUE TO SHEARING STRESS DISCONTINUITY IN AN ANISOTROPIC LAYER OVERLYING AN INITIALLY STRESSED ELASTIC HALF-SPACE
}

\author{
Santosh Kumar AND Dinbandhu Mandal
}

\begin{abstract}
The paper investigates the generation of SH-type waves due to a sudden application of a stress discontinuity which moves after creation at the anisotropic layer of finite thickness overlying an initially stressed isotropic half-space. The displacements are obtained in exact form by the method due to Cagniard modified by de Hoop. Two cases of shearing stress discontinuities are considered. The numerical results are obtained for a particular model and discussed by plotting graphs for displacement component with the elapsed time of the disturbance for different values of initial stress and also for different values of initial time at which pulses arrive.
\end{abstract}

\section{Introduction}

The notion of initial stress is essential to the study of seismic wave propagation. Biot [1940] is largely responsible for the notion's introduction and initial applications to elastic wave propagation; in [Biot 1965] he further developed the notion of initial stress. Many authors have used that book as fundamental to the study of wave propagation in an initially stressed medium. Abd-Alla and Ahmed [1999] analyzed Love waves propagation in a non-homogeneous orthotropic elastic layer under initial stress overlying semi-infinite medium. Khurana [2001] considered the effect of initial stress on the propagation of Love wave. Further significant steps were taken in [Das and Dey 1968; 1970, Dey 1971; Dey and Addy 1978; Chattopadhyay and De 1981; Chattopadhyay and Kar 1978; Majhi et al. 2016; 2017] to cite but a few works.

In addition to initial stress, shearing stress discontinuity also plays a vital role in the study of seismic wave propagation. Pal [1983] considered the problem of generation and propagation of SH-type waves due to non-uniformly moving stress

\section{Communicated by Holm Altenbach.}

MSC2010: 35L05, 74J15.

Keywords: SH-type waves, initial stress, shearing stress discontinuity, Cagniard-de Hoop technique. 
discontinuity in layered anisotropic elastic half-space using Garvin's [1956] techniques, which are a modification of Cagniard's [1939] technique. Pal and Kumar [2000] considered the generation of SH waves by a moving stress discontinuity in an anisotropic soil layer over an elastic half-space using the Cagniard-de Hoop special reduction technique [de Hoop 1960]. Next de Hoop [2002] considered the reflection and transmission properties of an elastic interfacial bonding of two semi-infinite solids, investigated for the simplest possible case of a line-source excited two-dimensional SH-wave. Pal and Mandal [2014] studied the generation of SH-type waves due to sudden application of a stress discontinuity which moves after creation at the sandy layer of finite thickness overlying an isotropic and inhomogeneous elastic half-space. Mandal et al. [2014] considered the disturbance and propagation of SH-type waves in an anisotropic soil layer overlying an inhomogeneous elastic half-space by a moving stress discontinuity. All authors have considered the effect of initial stress and shearing stress discontinuity separately but haven't considered the initial stress and shearing stress discontinuity together.

In the present problem our intention is to investigate the two dimensional problem of generation of SH-type waves at the free surface of an anisotropic layer due to an impulsive stress discontinuity moving with uniform velocity along the interface of initially stressed isotropic medium. The displacement is calculated numerically for two particular distances on the surface for two different types of the discontinuity in the shearing stress for different value of initial stress. It involves Laplace and Fourier transform and the inversion is based on Garvin's [1956] method. The problem discussed may be of importance in connection with the propagation of cracks in the layer. Two cases of stress discontinuity are considered and the numerical results are shown graphically.

\section{Formulation of problem}

We consider an anisotropic elastic layer of thickness $h$ with elastic constants $L_{1}, N_{1}$ and density $\rho_{1}$ over an initially stressed isotropic half-space with elastic constant $\mu_{2}$ and density $\rho_{2}$. The interface of these two media is considered at $z=0$ whereas free surface is at $z=-h$. Here, $z$ axis is directed vertically downward and $x$ axis is assumed in the direction of the propagation of wave with velocity $c$. For SH-type of waves the displacement does not depend on $y$ and if $(u, v, w)$ be the displacement at any point $P(x, y, z)$ into the medium then $u=w=0$ and $v$ are function of $x$, $z$ and $t$. The two equations of motion are identically satisfied. The geometrical configuration is depicted in Figure 1.

The equation of motion for the anisotropic layer (Medium I) without body forces is given by

$$
N_{1} \frac{\partial^{2} v_{1}}{\partial x^{2}}+L_{1} \frac{\partial^{2} v_{1}}{\partial z^{2}}=\rho_{1} \frac{\partial^{2} v_{1}}{\partial t^{2}}
$$




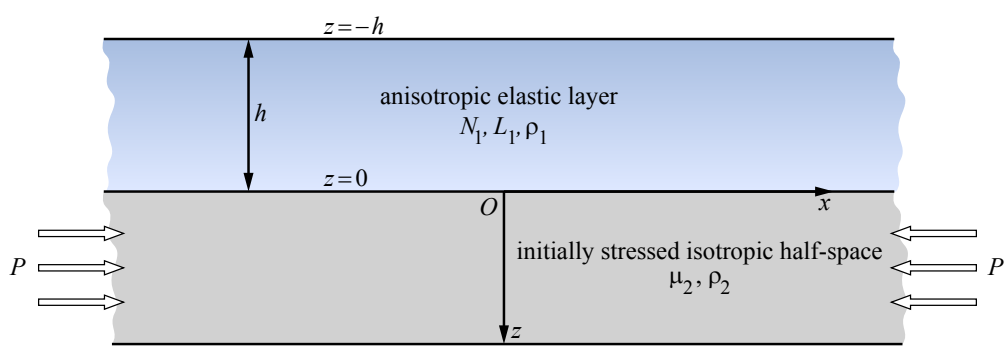

Figure 1. Geometry of problem.

The stress strain relation is given by $\left(\tau_{x y}\right)_{\mathrm{I}}=N_{1} \frac{\partial v_{1}}{\partial x}$ and $\left(\tau_{y z}\right)_{\mathrm{I}}=L_{1} \frac{\partial v_{1}}{\partial x}$.

The equation of motion for initially stressed isotropic half-space (Medium II) without body forces is given by

$$
\frac{\partial\left(\tau_{x y}\right)_{\mathrm{II}}}{\partial x}+\frac{\partial\left(\tau_{y z}\right)_{\mathrm{II}}}{\partial z}-\frac{P}{2} \frac{\partial v_{2}}{\partial x^{2}}=\frac{\partial^{2}}{\partial t^{2}}\left(\rho_{2} v_{2}\right) .
$$

The stress strain relation is given by $\left(\tau_{x y}\right)_{\mathrm{II}}=\mu_{2} \frac{\partial v_{2}}{\partial x}$ and $\left(\tau_{y z}\right)_{\mathrm{II}}=\mu_{2} \frac{\partial v_{2}}{\partial z}$. The boundary conditions are

$$
\begin{aligned}
\left(\tau_{y z}\right)_{\mathrm{I}} & =0 & & \text { at } z=-h, \\
v_{1} & =v_{2} & & \text { at } z=0, \\
\left(\tau_{y z}\right)_{\mathrm{I}}-\left(\tau_{y z}\right)_{\mathrm{II}} & =S(x, t) H(t) & & \text { at } z=0,
\end{aligned}
$$

where $S(x, t)$ is a function of $x$ and $t ; H(t)$ is the Heaviside unit function of time $t$.

\section{Method of solution}

The above problem can readily be solved by using the Laplace and Fourier transforms combined with the modified Cagniard-de Hoop [1960] method. The Laplace transform with respect to $t$ and the Fourier transform with respect to $x$ are defined by

$$
\widetilde{\bar{v}}=\int_{-\infty}^{\infty} e^{-i \xi x} d x \int_{0}^{\infty} e^{-p t} v(x, z ; t) d t .
$$

We can easily get the upper and lower layer with $v_{2} \rightarrow 0$ as $z \rightarrow \infty$ in the form

$$
\begin{aligned}
\bar{v}_{1}(x, z ; p) & =\int_{-\infty}^{\infty}\left(A \cosh s_{1} z+B \sinh s_{1} z\right) e^{i \xi x} d \xi, \\
\bar{v}_{2} & =\int_{-\infty}^{\infty} C e^{\left(i \xi x-s_{2} z\right)} d \xi
\end{aligned}
$$

where the constants $A, B$ and $C$ are to be determined from the boundary conditions 
(3)-(5):

$$
s_{1}=\left(\phi_{1}^{2} \xi^{2}+\frac{p^{2}}{\beta_{1}^{2}}\right)^{\frac{1}{2}}, \quad s_{2}=\left(\phi_{2}^{2} \xi^{2}+\frac{p^{2}}{\beta_{2}^{2}}\right)^{\frac{1}{2}},
$$

where $\beta_{1}^{2}=\frac{L_{1}}{\rho_{1}}, \beta_{2}^{2}=\frac{\mu_{2}}{\rho_{2}}, \phi_{1}^{2}=\frac{N_{1}}{L_{1}}$ and $\phi_{2}^{2}=1-\frac{P}{2 \mu_{2}}$.

It follows from the boundary conditions (3) and (4) that

$$
A=C, \quad A \cosh s_{1} h=B \sinh s_{1} h .
$$

Case I. Let

$$
S(x, t)= \begin{cases}Q, & a \leq x \leq b+V t \\ 0, & \text { elsewhere }\end{cases}
$$

where $Q$ is constant.

This definition of stress discontinuity shows that it is created in the region $x=a$ to $x=b$ and then expands with the uniform velocity $V$ in the $x$ direction. In particular, when $a=b=0$, the discontinuity is created at the origin and expands with uniform velocity $V$ in the $x$ direction.

From the boundary condition (5) one gets, with the help of (11),

$$
B L_{1} s_{1}+C \mu_{2} s_{2}=\frac{Q}{2 \pi p}\left[\frac{e^{-i \xi a}-e^{-i \xi b}}{i \xi}+\frac{e^{-i \xi b}}{i \xi+\frac{p}{V}}\right] .
$$

Solving for $A, B$ and $C$ from (10) and (12), we get the displacement function at the free surface at $z=-h$ in the form

$$
\begin{aligned}
& \bar{v}_{1}(x,-h ; p) \\
& =\frac{Q}{2 \pi p} \int_{-\infty}^{\infty} \frac{e^{i \xi x}}{\left(L_{1} s_{1} \sinh s_{1} h+\mu_{2} s_{2} \cosh s_{1} h\right)}\left[\frac{e^{-i \xi a}-e^{-i \xi b}}{i \xi}+\frac{e^{-i \xi b}}{i \xi+\frac{p}{V}}\right] d \xi \\
& =\frac{Q}{\pi p} \int_{-\infty}^{\infty} \frac{e^{\left(i \xi x-h s_{1}\right)}}{\left(L_{1} s_{1}+\mu_{2} s_{2}\right)}\left[\frac{e^{-i \xi a}-e^{-i \xi b}}{i \xi}+\frac{e^{-i \xi b}}{i \xi+\frac{p}{V}}\right]\left(1-K e^{-2 h s_{1}}\right)^{-1} d \xi
\end{aligned}
$$

where

$$
K=\frac{L_{1} s_{1}-\mu_{2} s_{2}}{L_{1} s_{1}+\mu_{2} s_{2}}<1
$$

represents the reflection coefficient of $\mathrm{SH}$-waves incident from the sandy medium at the interface between two half-spaces. The coefficients of different power of $K$ in series of (13) are associated with the pulses undergoing repeated reflection in the upper layer. Using the inverse Laplace transform, we can rewrite (13) in a convenient form:

$$
v_{1}(x,-h ; p)=L^{-1}\left(I_{1}+I_{2}+I_{3}\right)=L^{-1}\left(I_{1}\right)+L^{-1}\left(I_{2}\right)+L^{-1}\left(I_{3}\right),
$$


where $I_{1}, I_{2}$ and $I_{3}$ are defined in the Appendix. The inverse Laplace transforms of $I_{1}, I_{2}$ and $I_{3}$ are obtained by following [Garvin 1956]; details are in the Appendix.

Equation (15) gives the exact value of the surface displacement field $v_{1}(x,-h, t)$ surface.

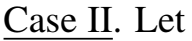

$$
S(x, t)=Q h \delta(x-V t),
$$

where $Q$ is a constant and $\delta(x-V t)$ is Dirac's delta function of argument $(x-V t)$. A term $h$ is included on the right-hand side of (16) so as to give $S$ as the dimension of a stress.

The boundary condition (5) gives

$$
B L_{1} s_{1}+C \mu_{2} s_{2}=\frac{Q h}{2 \pi V\left(i \xi+\frac{p}{V}\right)}
$$

Solving for $A, B$ and $C$ from (10) and (17) one gets

$$
\bar{v}_{1}(x,-h ; p)=\frac{Q h}{\pi V} \int_{-\infty}^{\infty} \frac{e^{\left(i \xi x-h s_{1}\right)}\left(1+K e^{-2 h s_{1}}+K^{2} e^{-4 h s_{1}}+\cdots\right)}{\left(i \xi+\frac{p}{V}\right)\left(L_{1} s_{1}+\mu_{2} s_{2}\right)} d \xi
$$

Proceeding similarly as in Case I we obtain

$$
v_{1}(x,-h, t)=\frac{2 Q \beta_{1} h}{\pi L_{1}} \sum_{n=1,3,5, \ldots} \int_{0}^{t} G_{n}\left[\zeta_{n}(\lambda)\right] d \lambda,
$$

where

$$
\begin{array}{r}
G_{n}\left[\zeta_{n}(t)\right]=\operatorname{Re}\left[\left\{1+\phi_{1}^{2} \zeta_{n}^{2}(t)\right\}^{\frac{1}{2}}+\mu\left(\varepsilon^{2}+\phi_{2}^{2} \zeta_{n}^{2}(t)\right)^{\frac{1}{2}}\right]^{-1} \times\left[\frac{\beta_{1}}{V}+i \zeta_{n}(t)\right]^{-1} \\
\times K^{\frac{n-1}{2}}\left[\zeta_{n}(t)\right] \frac{d \zeta_{n}(t)}{d t} H\left[t-\left(x^{2}+n^{2} h^{2}\right)^{\frac{1}{2}} \beta_{1}^{-1}\right]
\end{array}
$$

and

$$
\zeta_{n}(t)=\frac{\beta_{1}}{x^{2}+n^{2} h_{2} \phi_{1}^{2}}\left[i t x+n h\left\{t^{2}-\left(x^{2}+n^{2} h^{2} \phi_{1}^{2}\right) \beta_{1}^{-2}\right\}^{\frac{1}{2}}\right], \quad n=1,3,5, \ldots
$$

If the stress discontinuity is taken as $H(x)-H(x-V t)$ in place of $\delta(x-V t)$ the corresponding expression on the right-hand side of (18) will differ only by a constant factor from $I_{3}$ (with $a=b=0$ ). 


\section{Numerical results and discussion}

For numerical results, we have taken data for anisotropic layer and initially stressed half-space from [Babuska and Cara 1991]:

$$
\begin{aligned}
& N_{1}=175 \mathrm{GPa}, \quad L_{1}=202 \mathrm{GPa}, \quad \rho_{1}=4408 \mathrm{~kg} / \mathrm{m}^{3} \\
& \mu_{2}=91.6 \mathrm{GPa}, \quad \rho_{2}=3582 \mathrm{~kg} / \mathrm{m}^{3} .
\end{aligned}
$$

The values of $K_{1} v_{1}(x,-h, t)$ for $x=7 h$ and $x=14 h$ have been plotted against $\tau_{1}=\tau-\tau_{0}$, where $\tau_{0}$ denotes the time at which the disturbance arrives at the point of observation with $K_{1}=\frac{\pi L_{1}}{2 Q \beta_{1} h}, \tau=\frac{t \beta_{1}}{h}$ is the time of the disturbance to arrive from source to initial point. The value of $\tau$ at $x=7 h$ is $\left(7^{2}+n^{2} \phi_{1}^{2}\right)^{1 / 2}, n=1,3,5, \ldots$ and $\tau_{0}=7.06$ at $n=1$ and the value of $\tau$ at $x=14 h$ is $\left(14^{2}+n^{2} \phi_{1}^{2}\right)^{1 / 2}, n=1,3,5, \ldots$ and $\tau_{0}=14.03$ at $n=1$.

When $x=7 h$, for six initial values, we have

$$
K_{1} v_{1}(x,-h, t)=\sum_{n=1,3,5,7,9,11} A^{0}\left(\theta_{n}\right) \cosh ^{-1}\left(\tau / \sqrt{7^{2}+n^{2} \phi_{1}^{2}}\right) H\left(\tau-\sqrt{7^{2}+n^{2} \phi_{1}^{2}}\right) \text {, }
$$

where

$$
A^{0}\left(\theta_{n}\right)=\operatorname{Re} \frac{\left[\left(1-\phi_{1}^{2} \cos ^{2} \theta_{n}\right)^{\frac{1}{2}}-\mu\left(\varepsilon^{2}-\phi_{2}^{2} \cos ^{2} \theta_{n}\right)^{\frac{1}{2}}\right]^{\frac{n-1}{2}} \sin \theta_{n}}{\left[\left(1-\phi_{1}^{2} \cos ^{2} \theta_{n}\right)^{\frac{1}{2}}+\mu\left(\varepsilon^{2}-\phi_{2}^{2} \cos ^{2} \theta_{n}\right)^{\frac{1}{2}}\right]^{\frac{n-3}{2}}\left(\frac{\beta_{1}}{V}-\cos \theta_{n}\right)} ;
$$

represents the reflection coefficient of SH-type waves incident from the anisotropic medium to initially stressed isotropic half-space. When $x=14 h$, for six initial values, we have the same expression for $K_{1} v_{1}(x,-h, t)$, with $7^{2}$ replaced by $14^{2}$.

Figures 2 and 3 show graphs of the variation of displacement with elapsed time $\tau_{1}$ for different values of initial stress and for different values of initial time at which pulses arrive.

In Figure 2, top, the graph is plotted for the disturbance effect for $x=7 \mathrm{~h}$ and $x=14 h$ and fixed value of $P=1 \mathrm{GPa}$. From the figure it can be observed that all the curves start from the origin with sharp changes in their slope and after sometime the curves get smooth. Also, it reflects that the disturbance is more prominent for early arrival of pulses i.e. for $x=7 h$ has more jumping effect than for $x=14 h$. If the place of observation has more distance from the source the impact of pluses is less. In Figure 2, bottom, the graph is plotted for the disturbance effect for $x=7 \mathrm{~h}$ and $x=14 h$ and fixed value of $P=10 \mathrm{GPa}$. The nature of the curves remains the same, but the magnitude of disturbance increases to a large extent as initial stress increases.

In Figure 3, top, the graph is plotted for the disturbance for $x=7 \mathrm{~h}$ and different values of $P(1 \mathrm{GPa}, 10 \mathrm{GPa}$ and $100 \mathrm{GPa})$. The nature of the curves is oscillating 

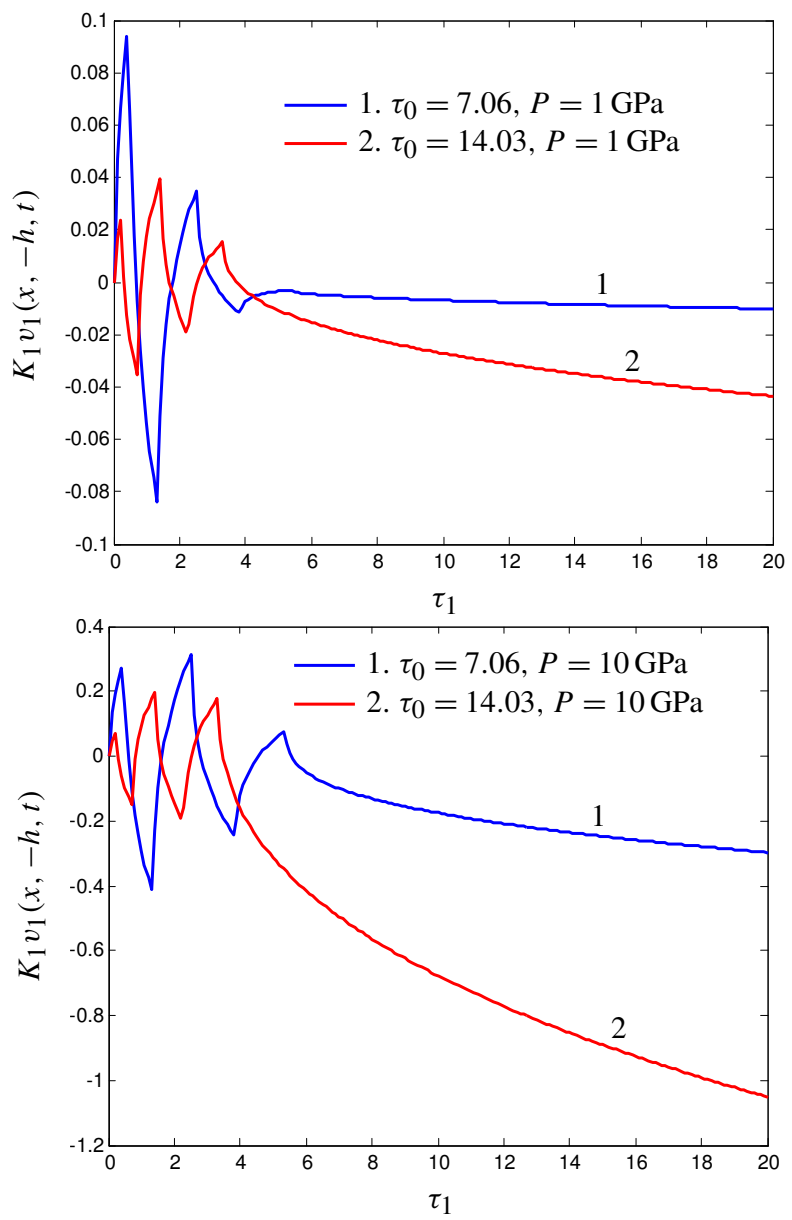

Figure 2. Variation of $K_{1} v_{1}(x,-h, t)$ with $\tau_{1}$ for $x=7 h$ and $x=14 h$, for $P=1 \mathrm{GPa}$ (top) and $P=10 \mathrm{GPa}$.

and after some time the curves become smooth and steady. As we increase the values of initial stress, the jumping effect increases. In Figure 3, bottom, the graph is plotted for the disturbance for $x=14 h$ and the same values of $P$. The nature of the curves remains the same, but the effect of disturbance is reduced to a large extent due to the late arrival of the pulses.

\section{Conclusions}

The generation of SH-type waves at the free surface of an anisotropic layer due to an impulsive stress discontinuity moving with uniform velocity along the interface of initially stressed isotropic medium has been considered. The displacement is calculated numerically for two particular distances on the surface for two different 

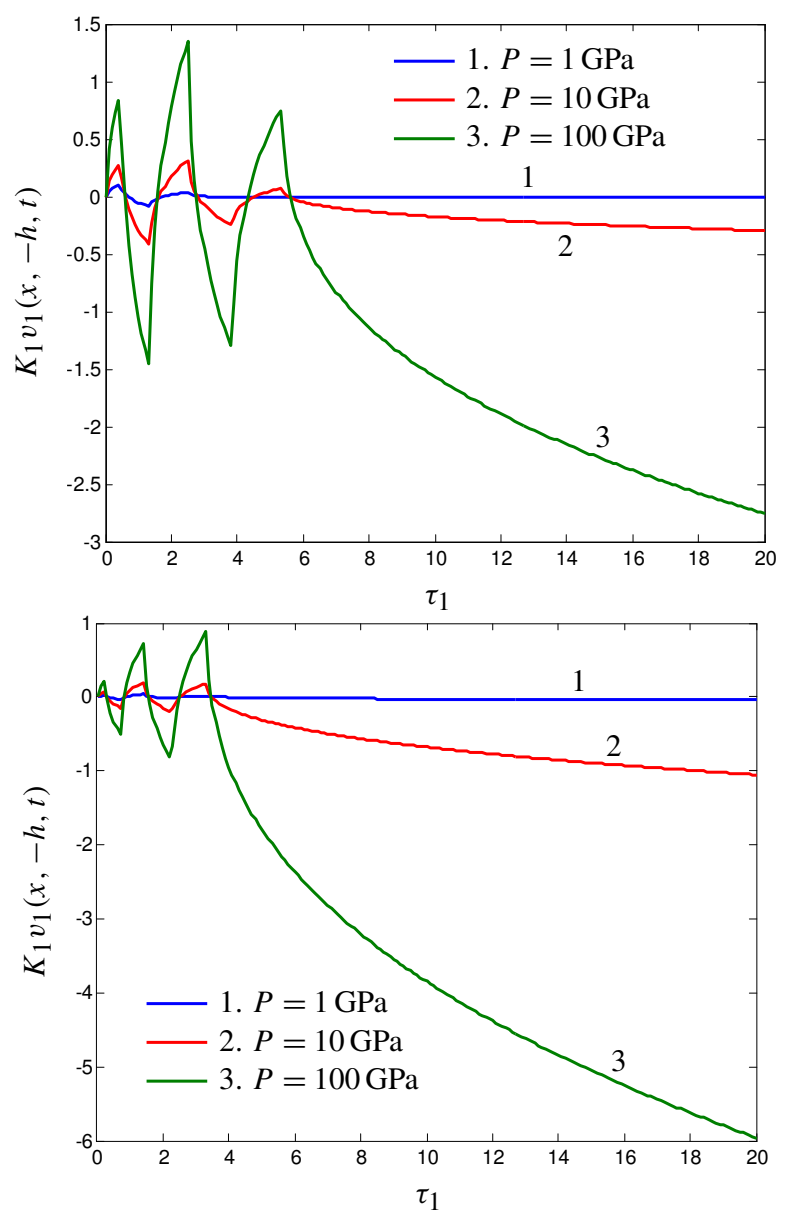

Figure 3. Variation of $K_{1} v_{1}(x,-h, t)$ with $\tau_{1}$ for $x=7 h$ (top) and $x=14 h$ (bottom) for different values of $P$.

types of the discontinuity in the shearing stress for different value of initial stress. It involves Laplace and Fourier transform and the inversion is based on Garvin's [1956] method. The numerical results are obtained for a particular model. From the figures it can be observed that initial stress and initial time at which pulses arrive has a significant effect. From the graph it is visible that that the displacement factor starts oscillating and after sometime it gets stable. The results are more comprising with the real scenario as we see that the disturbance arrives at the surface, it shakes the surface for a while and slowly gets stable. Also, from the figures it is visible that if the observer is nearer to the source then pulses arrive early and produce more disturbance and if the medium is highly pre-stressed then it produces more disturbance. 


\section{Appendix}

We have

$$
\begin{aligned}
& I_{1}=\frac{Q}{\pi p} \int_{-\infty}^{\infty} \frac{\left(1-K e^{-2 h s_{1}}\right)^{-1}}{i \xi\left(L_{1} s_{1}+\mu_{2} s_{2}\right)} e^{\left(i \xi x_{1}-h s_{1}\right)} d \xi, \\
& I_{2}=\frac{Q}{\pi p} \int_{-\infty}^{\infty} \frac{\left(1-K e^{-2 h s_{1}}\right)^{-1}}{i \xi\left(L_{1} s_{1}+\mu_{2} s_{2}\right)} e^{\left(i \xi x_{2}-h s_{1}\right)} d \xi, \\
& I_{3}=\frac{Q}{\pi p} \int_{-\infty}^{\infty} \frac{\left(1-K e^{-2 h s_{1}}\right)^{-1}}{\left(i \xi+\frac{p}{V}\right)\left(L_{1} s_{1}+\mu_{2} s_{2}\right)} e^{\left(i \xi x_{2}-h s_{1}\right)} d \xi,
\end{aligned}
$$

with $x_{1}=x-a$ and $x_{2}=x-b$. In order to evaluate the Laplace inversion integral, we have used Garvin's method; see [Garvin 1956] for discussion of the contour integration and mapping.

Next for non-dimensionalisation, we substitute $\xi=\frac{\zeta p}{\beta_{1}}, \mu=\frac{\mu_{2}}{L_{1}}, \frac{\beta_{1}}{\beta_{2}}=\varepsilon$ in the integral above so that $s_{1}=\frac{p}{\beta_{1}}\left(1+\phi_{1}^{2} \zeta^{2}\right)^{\frac{1}{2}}, s_{2}=\frac{p}{\beta_{2}}\left(\varepsilon^{2}+\phi_{2}^{2} \zeta^{2}\right)^{\frac{1}{2}}$ and

$$
K=\frac{\left(1+\phi_{1}^{2} \zeta^{2}\right)^{\frac{1}{2}}-\mu\left(\varepsilon^{2}+\phi_{2}^{2} \zeta^{2}\right)^{\frac{1}{2}}}{\left(1+\phi_{1}^{2} \zeta^{2}\right)^{\frac{1}{2}}+\mu\left(\varepsilon^{2}+\phi_{2}^{2} \zeta^{2}\right)^{\frac{1}{2}}} .
$$

Thus we obtain

$$
I_{1}=\frac{2 Q}{\pi p} \operatorname{Im} \int_{0}^{\infty} \frac{e^{\left(i \xi x_{1}-h s_{1}\right)}\left(1+K e^{-2 h s_{1}}+K^{2} e^{-4 h s_{1}}+\cdots\right)}{\xi\left(L_{1} s_{1}+\mu_{2} s_{2}\right)} d \xi .
$$

The first term in $I_{1}$ is

$$
I_{1,1}=\frac{2 Q \beta_{1}}{\pi p L_{1}} \operatorname{Im} \int_{0}^{\infty} \frac{\exp \left[-p\left\{-i \zeta x_{1}+h\left(1+\phi_{1}^{2} \zeta^{2}\right)^{\frac{1}{2}}\right\} / \beta_{1}\right]}{p \zeta\left[\left(1+\phi_{1}^{2} \zeta^{2}\right)^{\frac{1}{2}}+\mu\left(\varepsilon^{2}+\phi_{2}^{2} \zeta^{2}\right)^{\frac{1}{2}}\right]} d \zeta .
$$

The integrand (A.2) has singularities at $\zeta=0, \pm \frac{i}{\phi_{1}}, \pm \frac{i \varepsilon}{\phi_{2}}$. Let $t=\left\{-i \zeta x_{1}+h(1+\right.$ $\left.\left.\phi_{1}^{2} \zeta^{2}\right)^{\frac{1}{2}}\right\} / \beta_{1}$. Then by inversion $\zeta(t)=\frac{\beta_{1}}{x_{1}^{2}+\phi_{1}^{2} h^{2}}\left[i t x_{1}+h\left\{t^{2}-\left(x_{1}^{2}+\phi_{1}^{2} h^{2}\right) \beta_{1}^{-2}\right\}^{\frac{1}{2}}\right]$. The mapping of the $\zeta$-plane into the $t$-plane is shown in Figure 4.

Making the reference to the Figure 4 and the paper of [Pal 1983], we find

$$
L^{-1} I_{1,1}=\frac{2 Q \beta_{1}}{\pi L_{1}} \int_{0}^{t}\left(t-\lambda_{1}\right) G_{1,1}\left[\zeta_{1,1}\left(\lambda_{1}\right)\right] d \lambda_{1},
$$

where $L[t H(t)]=\frac{1}{p^{2}}$ and

$$
\begin{aligned}
G_{1,1}\left[\zeta_{1,1}(t)\right]= & \operatorname{Im}\left[\left(1+\phi_{1}^{2} \zeta_{1,1}^{2}\right)^{\frac{1}{2}}+\mu\left(\varepsilon^{2}+\phi_{2}^{2} \zeta_{1,1}^{2}\right)^{\frac{1}{2}}\right]^{-1} \zeta_{1,1}^{-1}(t) \\
& \times \frac{d \zeta_{1,1}(t)}{d t} H\left[t-\left\{\eta\left(x_{1}^{2}+h^{2} \phi_{1}^{2}\right)\right\}^{\frac{1}{2}} \beta_{1}^{-1}\right]
\end{aligned}
$$




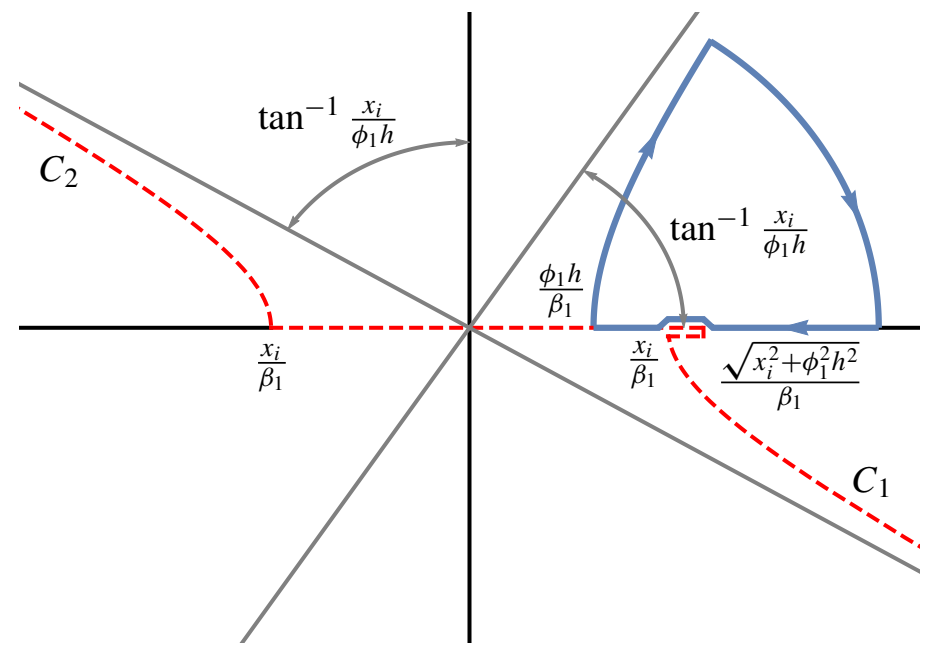

Figure 4. The $t$-plane showing the mapping and the contour of integration.

Since for $\frac{\phi_{1} h}{\beta_{1}}<t<\frac{\left(x_{1}^{2}+h^{2} \phi_{1}^{2}\right)^{\frac{1}{2}}}{\beta_{1}},\left[\left(1+\phi_{1}^{2} \zeta_{1,1}^{2}\right)^{\frac{1}{2}}+\mu\left(\varepsilon^{2}+\phi_{2}^{2} \zeta_{1,1}^{2}\right)^{\frac{1}{2}}\right] \zeta_{1,1}^{-1}(t) \times \frac{d \zeta_{1,1}(t)}{d t}$ is real. In general,

$$
L^{-1} I_{1, n}=\frac{2 Q \beta_{1}}{\pi L_{1}} \int_{0}^{t}\left(t-\lambda_{1}\right) G_{1, n}\left[\zeta_{1, n}\left(\lambda_{1}\right)\right] d \lambda_{1},
$$

where

$$
\begin{aligned}
G_{1, n}\left[\zeta_{1, n}(t)\right]= & \operatorname{Im}\left[\left\{1+\phi_{1}^{2} \zeta_{1, n}^{2}(t)\right\}^{\frac{1}{2}}+\mu\left(\varepsilon^{2}+\phi_{2}^{2} \zeta_{1, n}^{2}(t)\right)^{\frac{1}{2}}\right]^{-1} \zeta_{1, n}^{-1}(t) \\
& \times K^{\frac{n-1}{2}}\left[\zeta_{1, n}(t)\right] \frac{d \zeta_{1, n}(t)}{d t} H\left[t-\left\{\left(x_{1}^{2}+h^{2} \phi_{1}^{2}\right)\right\}^{\frac{1}{2}} \beta_{1}^{-1}\right],
\end{aligned}
$$

and

$$
\zeta_{1, n}(t)=\frac{\beta_{1}}{x_{1}^{2}+n^{2} h^{2} \phi_{1}^{2}}\left[i t x_{1}+n h\left\{t^{2}-\left(x_{1}^{2}+n^{2} h^{2} \phi_{1}^{2}\right) \beta_{1}^{-2}\right\}^{\frac{1}{2}}\right], \quad n=1,3,5, \ldots
$$

So that

$$
L^{-1} I_{1}=\sum_{n=1,3,5, \ldots} L^{-1} I_{1, n}
$$

Similarly

$$
L^{-1} I_{2}=\sum_{n=1,3,5, \ldots} L^{-1} I_{2, n}
$$

where $x_{1}$ is replaced by $x_{2}$. 
Proceeding in the same way, we get

$$
L^{-1} I_{3}=\sum_{n=1,3,5, \ldots} L^{-1} I_{3, n}
$$

where

$$
L^{-1} I_{3, n}=\frac{2 Q \beta_{1}}{\pi L_{1}} \int_{0}^{t}\left(t-\lambda_{1}\right) G_{3, n}\left[\zeta_{2, n}\left(\lambda_{1}\right)\right] d \lambda_{1}
$$

$$
\begin{aligned}
G_{3, n}\left[\zeta_{2, n}(t)\right]= & \operatorname{Re}\left[\left\{1+\phi_{1}^{2} \zeta_{2, n}^{2}(t)\right\}^{\frac{1}{2}}+\mu\left(\varepsilon^{2}+\phi_{2}^{2} \zeta_{2, n}^{2}(t)\right)^{\frac{1}{2}}\right]^{-1} \times\left[\frac{\beta_{1}}{V}+i \zeta_{2, n}(t)\right]^{-1} \\
& \times K^{\frac{n-1}{2}}\left[\zeta_{2, n}(t)\right] \frac{d \zeta_{2, n}(t)}{d t} H\left[t-\left(x_{2}^{2}+n^{2} h^{2} \phi_{1}^{2}\right)^{\frac{1}{2}} \beta_{1}^{-1}\right]
\end{aligned}
$$

and $\zeta_{2, n}(t)$ is given by Appendix with $x_{2}$ in place of $x_{1}$.

\section{Acknowledgement}

Kumar, is grateful to the I.S.M., Dhanbad for Financial support in the form of a Research Fellowship during Ph.D. and to my guide Prof. P. C. Pal for their immense support and guidance. The authors declare that they have no conflict of interest.

\section{References}

[Abd-Alla and Ahmed 1999] A. M. Abd-Alla and S. M. Ahmed, "Propagation of Love waves in a non-homogeneous orthotropic elastic layer under initial stress overlying semi-infinite medium", Appl. Math. Comput. 106:2 (1999), 265-275.

[Babuska and Cara 1991] V. Babuska and M. Cara, Seismic anisotropy in the earth, Kluwer, Dordrecht, 1991.

[Biot 1940] M. A. Biot, "The influence of initial stress on elastic wave", J. Appl. Phys. 8 (1940), 522-530.

[Biot 1965] M. A. Biot, Mechanics of incremental deformation, Wiley, New York, 1965.

[Cagniard 1939] L. Cagniard, Reflection et refraction des ondes seismiques progressive, GauthierVillars, Paris, 1939.

[Chattopadhyay and De 1981] A. Chattopadhyay and R. K. De, "Propagation of Love type waves in a visco elastic initially stressed layer overlying a visco-elastic half-space with irregular interface", Rev. Roum. Sci. Tech. Mech. Appl. 26:3 (1981), 449-460.

[Chattopadhyay and Kar 1978] A. Chattopadhyay and B. K. Kar, "On the dispersion curves of Love types waves in an initially stressed crustal layer having an irregular interface", Geophys. Res. Bull. 16:1 (1978), 13-23.

[Das and Dey 1968] S. C. Das and S. Dey, "Note on gravity waves", Indian J. Engin. Math. 1:2 (1968), 155-160.

[Das and Dey 1970] S. C. Das and S. Dey, "Edge wave under initial stress", Appl. Sci. Res. 22:1 (1970), 382-389.

[de Hoop 1960] A. T. de Hoop, "A modification of Cagniard's method for solving seismic pulse problem”, Appl. Sci. Res. B 8:1 (1960), 349-356. 
[de Hoop 2002] A. T. de Hoop, "Reflection and transmission of a transient, elastic, line-source excited SH wave by a planer, elastic bonding surface in solid", Int. J. Solids Struct. 39:21-22 (2002), 5379-5391.

[Dey 1971] S. Dey, "Wave propagation in two layered medium under initial stresses", J. Pure Appl. Geophys. (Switzerland) 90:1 (1971), 38-52.

[Dey and Addy 1978] S. Dey and S. K. Addy, "Love waves under initial stresses in a visco-elastic medium overlying an elastic half-space”, Gerlands Beiträge Geophys. 87:4 (1978), 306-311.

[Garvin 1956] W. W. Garvin, "Exact transient solution of the Buried line source problem", Proc. R. Soc. Lond. A 234:1199 (1956), 528-541.

[Khurana 2001] P. Khurana, "Love wave propagation in a prestressed medium", Indian J. Pure Appl. Math. 32:8 (2001), 1201-1207.

[Majhi et al. 2016] S. Majhi, P. C. Pal, and S. Kumar, "Love waves in a layered functionally graded piezoelectric structure under initial stress”, Waves Random Complex Media 26 (2016), 535-552.

[Majhi et al. 2017] S. Majhi, P. C. Pal, and S. Kumar, "Reflection and transmission of plane SHwaves in an initially stressed inhomogeneous anisotropic magnetoelastic medium", J. Seismolog. 21 (2017), 155-163.

[Mandal et al. 2014] P. C. Pal, D. Mandal, and S. Kumar, "Disturbance of SH-type waves due to moving stress discontinuity in an anisotropic soil layer overlying an inhomogeneous elastic halfspace”, Sadhana 39:2 (2014), 451-465.

[Pal 1983] P. C. Pal, "Generation of SH-type waves due to non-uniformly moving stress-discontinuity in layered anisotropic elastic half-space”, Acta Mech. 49:3-4 (1983), 209-220.

[Pal and Kumar 2000] P. C. Pal and L. Kumar, "Generation of SH waves by a moving stress discontinuity in an anisotropic soil layer over an elastic half-space", Acta Geophys. 48:4 (2000), 465-478.

[Pal and Mandal 2014] P. C. Pal and D. Mandal, "Generation of SH-type waves due to shearing stress discontinuity in a sandy layer overlying an isotropic and inhomogeneous elastic half-space", Acta Geophys. 62:1 (2014), 44-58.

Received 21 Oct 2017. Revised 9 Feb 2018. Accepted 26 Apr 2018.

SANTOSH KUMAR: santosh453@gmail.com

Department of Mathematics, VVIT, Vidya Vihar Institute of Technology, Purnea 854303, India

DinBandhu MANDAL: dinbandhumandal@gmail.com

Department of Mathematics, Chaibasa Engineering College, West Singhbhum 833215, India 
EDITORIAL BOARD

ANTONIO CARCATERRA

ERIC A. CARLEN

FRANCESCO DELL'ISOLA

RAFFAELE ESPOSITO

ALBERT FANNJIANG

Gilles A. FranCFORT

Pierangelo MARCATI

JEAN-JACQUES MARIGO

PETER A. MARKOWICH

MARTIN OSTOJA-STARZEWSKI

PIERRE SEPPECHER

DAVID J. STEIGMANN

PAUl STEINMANN

PierRe M. SuQueT

MANAGING EDITORS

MICOL AMAR

CORRADO LATTANZIO

ANGELA MADEO

MARTIN OSTOJA-STARZEWSKI

ADVISORY BOARD

ADNAN AKAY

Holm AltenBaCH

MICOL AMAR

HARM ASKES

TEODOR ATANACKOVIĆ

VICTOR BERDICHEVSKY

GUY BOUCHITTÉ

ANDREA BRAIDES

ROBERTO CAMASSA

MAURO CARFORE

ERIC DARVE

FELIX DARVE

ANNA DE MASI

GianPiEtro DEL Piero

EMMANUELE Di BENEDETTO

BERNOLD FIEDLER

IRENE M. GAMBA

DAVID Y. GAO

SERGEY GAVRILYUK

TIMOTHY J. HEALEY

DOMINIQUE JEULIN

ROGER E. KHAYAT

CORRADO LATTANZIO

ROBERT P. LIPTON

ANGELO LUONGO

ANGELA MADEO

JUAN J. MANFREDI

CARLO MARCHIORO

GÉRARD A. MAUGIN

ROBERTO NATALINI PATRIZIO NEFF

ANDREY PIATNITSKI

ERRICO PRESUTTI

MARIO PULVIRENTI

LUCIO RUSSO

Miguel A. F. SANJUAN

PATRICK SElVADURAI

ALEXANDER P. SEYRANIAN

MIROSLAV ŠILHAVÝ

GUIDO SWEERS

ANTOINETTE TORDESILLAS

LEV TRUSKINOVSKY

JUAN J. L. VELÁZQUEZ VINCENZO VESPRI ANGELO VULPIANI msp.org/memocs

Università di Roma "La Sapienza", Italia

Rutgers University, USA

(CO-CHAIR) Università di Roma "La Sapienza", Italia

(TREASURER) Università dell'Aquila, Italia

University of California at Davis, USA

(CO-CHAIR) Université Paris-Nord, France

Università dell'Aquila, Italy

École Polytechnique, France

DAMTP Cambridge, UK, and University of Vienna, Austria

(CHAIR MANAGING EDITOR) Univ. of Illinois at Urbana-Champaign, USA

Université du Sud Toulon-Var, France

University of California at Berkeley, USA

Universität Erlangen-Nürnberg, Germany

LMA CNRS Marseille, France

Università di Roma "La Sapienza", Italia

Università dell'Aquila, Italy

Université de Lyon-INSA (Institut National des Sciences Appliquées), France

(CHAIR MANAGING EDITOR) Univ. of Illinois at Urbana-Champaign, USA

Carnegie Mellon University, USA, and Bilkent University, Turkey

Otto-von-Guericke-Universität Magdeburg, Germany

Università di Roma "La Sapienza", Italia

University of Sheffield, UK

University of Novi Sad, Serbia

Wayne State University, USA

Université du Sud Toulon-Var, France

Università di Roma Tor Vergata, Italia

University of North Carolina at Chapel Hill, USA

Università di Pavia, Italia

Stanford University, USA

Institut Polytechnique de Grenoble, France

Università dell'Aquila, Italia

Università di Ferrara and International Research Center MEMOCS, Italia

Vanderbilt University, USA

Freie Universität Berlin, Germany

University of Texas at Austin, USA

Federation University and Australian National University, Australia

Université Aix-Marseille, France

Cornell University, USA

École des Mines, France

University of Western Ontario, Canada

Università dell' Aquila, Italy

Louisiana State University, USA

Università dell'Aquila, Italia

Université de Lyon-INSA (Institut National des Sciences Appliquées), France University of Pittsburgh, USA

Università di Roma "La Sapienza”, Italia

Université Paris VI, France

Istituto per le Applicazioni del Calcolo "M. Picone", Italy

Universität Duisburg-Essen, Germany

Narvik University College, Norway, Russia

Università di Roma Tor Vergata, Italy

Università di Roma "La Sapienza”, Italia

Università di Roma “Tor Vergata”, Italia

Universidad Rey Juan Carlos, Madrid, Spain

McGill University, Canada

Moscow State Lomonosov University, Russia

Academy of Sciences of the Czech Republic

Universität zu Köln, Germany

University of Melbourne, Australia

École Polytechnique, France

Bonn University, Germany

Università di Firenze, Italia

Università di Roma La Sapienza, Italia

MEMOCS (ISSN 2325-3444 electronic, 2326-7186 printed) is a journal of the International Research Center for the Mathematics and Mechanics of Complex Systems at the Università dell'Aquila, Italy.

Cover image: "Tangle” by $\odot$ John Horigan; produced using the Context Free program (contextfreeart.org).

PUBLISHED BY

7 mathematical sciences publishers

nonprofit scientific publishing

http://msp.org/

(C) 2018 Mathematical Sciences Publishers 
Mathematics and Mechanics of Complex Systems vol. 6 no. 3

The variational structure of classical plasticity

Gianpietro Del Piero

Far-reaching Hellenistic geographical knowledge hidden in

Ptolemy's data

\section{Lucio Russo}

Generation of SH-type waves due to shearing stress discontinuity in an anisotropic layer overlying an initially stressed elastic half-space

Santosh Kumar and Dinbandhu Mandal

Strain gradient and generalized continua obtained by homogenizing frame lattices

Houssam Abdoul-Anziz and Pierre Seppecher

On the effect of phase transition on the manifold dimensionality: application to the Ising model

Elena Lopez, Adrien Scheuer, Emmanuelle Abisset-Chavanne and Francisco Chinesta

MEMOCS is a journal of the International Research Center for the Mathematics and Mechanics of Complex Systems at the Università dell' Aquila, Italy.

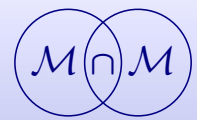

